\title{
Characterizing a full spectrum of physico-chemical properties of (20S)- and (20R)-ginsenoside Rg3 to be proposed as standard reference materials
}

\author{
Il-Woung Kim ${ }^{1}$, Won Suk Sun ${ }^{2}$, Bong-Sik Yun ${ }^{3}$, Na-Ri Kim ${ }^{1}$, Dongsun Min ${ }^{2}$, and Si-Kwan Kim ${ }^{{ }^{*}}$ \\ ${ }^{1}$ Department of Life Science, College of Biomedical and Health Science, Konkuk University, Chungju 380-701, Korea \\ ${ }^{2}$ SK Chemicals, Seongnam 463-400, Korea \\ ${ }^{3}$ Division of Biotechnology, College of Environmental and Bioresource Sciences, Chonbuk National University, Jeonju 561- \\ 756, Korea
}

The authentication of the physico-chemical properties of ginsenosides reference materials as well as qualitative and quantitative batch analytical data based on validated analytical procedures is a prerequisite for certifying good manufacturing practice (GMP). Ginsenoside $\mathrm{Rb} 1$ and $\mathrm{Rg} 1$, representing protopanaxadiol and protopanaxatriol ginsenosides, respectively, are accepted as marker substances in quality control standards worldwide. However, the current analytical methods for these two compounds recommended by Korean, Chinese, European, and Japanese pharmacopoeia do not apply to red ginseng preparations, particularly the extract, because of the relatively low content of the two agents in red ginseng compared to white ginseng. In manufacturing fresh ginseng into red ginseng products, ginseng roots are exposed to a high temperature for many hours, and the naturally occurring ginsenoside $\mathrm{Rb} 1$ and $\mathrm{Rg} 1$ are converted to artifact ginsenosides such as $\mathrm{Rg} 3, \mathrm{Rg} 5, \mathrm{Rh} 1$, and $\mathrm{Rh} 2$ during the heating process. The analysis of ginsenosides in commercially available ginseng products in Korea led us to propose the inclusion of the (20S)- and (20R)-ginsenoside $\mathrm{Rg} 3$, including ginsenoside $\mathrm{Rb} 1$ and $\mathrm{Rg} 1$, as additional reference materials for ginseng preparations. (20S)- and (20R)-ginsenoside Rg3 were isolated by Diaion HP-20 adsorption chromatography, silica gel flash chromatography, recrystallization, and preparative HPLC. HPLC fractions corresponding to those two ginsenosides were recrystallized in appropriate solvents for the analysis of physico-chemical properties. Documentation of those isolated ginsenosides was achieved according to the method proposed by Gaedcke and Steinhoff. The ginsenosides were subjected to analyses of their general characteristics, identification, purity, content quantification, and mass balance tests. The isolated ginsenosides showed 100\% purity when determined by the three HPLC systems. Also, the water content was found to be $0.534 \%$ for $(20 S)-\operatorname{Rg} 3$ and $0.920 \%$ for $(20 R)-\operatorname{Rg} 3$, meaning that the net mass balances for (20S)-Rg3 and (20R)-Rg3 were $99.466 \%$ and $99.080 \%$, respectively. From these results, we could assess and propose a full spectrum of physico-chemical properties of $(20 S)$ - and (20R)-ginsenoside $\mathrm{Rg} 3$ as standard reference materials for GMP-based quality control.

Keywords: Panax ginseng, (20S)- and (20R)-ginsenoside Rg3, Physico-chemical property, Standard reference material, Documentation

\section{INTRODUCTION}

Panax ginseng has been used in traditional medicine in East Asian countries for over two thousand years. The

(c) This is an Open Access article distributed under the terms of the Creative Commons Attribution Non-Commercial License (http://creativecommons.org/licenses/by-nc/3.0/) which permits unrestricted non-commercial use, distribution, and reproduction in any medium, provided the original work is properly cited. empirical, evidence-based clinical efficacies of ginseng have been elucidated by modern and multi-disciplinary

Received 29 Jul. 2012, Revised 17 Oct. 2012, Accepted 18 Oct. 2012

*Corresponding author

E-mail: skkim@kku.ac.kr

Tel: +82-43-840-3574, Fax: +82-2-43-840-3929 
experiments and thus enabled us to find a wide spectrum of pharmacological effects: antifatigue, immunomodulatory therapy, facilitation of blood circulation, spermatogenesis stimulation, and antistress effects are just a few of many [1]. To date, saponins (ginsenosides), polysaccharides, and polyacetylenes have been characterized from red or white $P$. ginseng as the active therapeutic ingredients $[2,3]$. However, ginsenosides are regarded as the major active components of ginseng among those bioactive agents and are therefore employed as the reference material for quality control. A total of 38 ginsenosides have been isolated and characterized from the ginseng plant [4].

Ginseng is classified as an herbal medicine or health food supplement depending on the country. The United States and Korea categorize it as a health food supplement, but the World Health Organization (WHO), European Union (EU), Middle East, and India consider it to be medicine. To market ginseng products in the EU, we need marketing authorization. Completion of the common technical document (CTD) is essential paper work for applying to the marketing authorization holder (MAH). Good manufacturing practice (GMP)-based quality control is an integral part of the CTD. An authenticity document of the physico-chemical properties for ginsenosides reference materials (RM) and both qualitative and quantitative batch analytical data based on validated analytical procedures are prerequisites to certify GMP. In this respect, it can be said that GMP will not be possible without an authenticity document for the full spectrum of physico-chemical properties of the reference materials. In addition, it is obligatory that every quality analytical method be validated according to guidelines issued by the International Conference on Harmonization (ICH) [5]. Japan, Korea, and European countries require quantitative analytical HPLC data for the ginsenoside $\mathrm{Rb} 1$ and $\mathrm{Rg} 1$ and representative reference materials for the protopanaxadiol and protopanaxatriol ginsenoside. However, the current analytical methods for these two compounds recommended by the Korean, Chinese, European, and Japanese pharmacopoeia are not applicable to red ginseng preparations, particularly the extract, because of the relatively lower content of these two agents in red ginseng compared to white ginseng. In the manufacturing process of fresh ginseng into red ginseng products, ginseng roots are exposed to high temperatures for many hours, and the naturally occurring $\mathrm{Rb} 1$ and $\mathrm{Rg} 1$ are converted to artifact ginsenosides such as $\mathrm{Rg} 3, \mathrm{Rg} 5$, $\mathrm{Rh} 1$, and Rh2, during the heating process. An analysis of ginseng extracts available in the Korean market led us to include (20S)- and (20R)-ginsenoside Rg3, including ginsenoside $\mathrm{Rb} 1$ and $\mathrm{Rg} 1$, as additional reference materials for ginseng preparations. Moreover, methods for authenticity documentation of ginsenoside RM are not available from monographs of the WHO's selected medicinal plants and Japanese and Chinese pharmacopoeias. Therefore, the CTD and ICH guideline-based application is not strictly required for the application format of $\mathrm{MAH}$ in Japan and China. However, the EU requires a medicinal level of quality assurance data for ginseng preparations. In addition, regulation of quality control is gradually becoming stricter in China as well as in Korea due to adulterations and low-quality herbal medicinal products.

Germany has a well-established scientific and regulatory basis for development, quality assurance, and marketing authorization. We could find detailed authenticity documentation methods for RM from a textbook [6] written by German experts in drug regulatory affairs. We have thus far isolated 13 ginsenosides, including (20S)and (20R)-ginsenoside $\mathrm{Rg} 3$, in their highest purity by employing adsorption chromatography, silica gel column chromatography, preparative HPLC and recrystallization; authenticity documentation for the isolated ginsenosides was achieved. Among those ginsenosides, documentation for the (20S)- and (20R)-ginsenoside Rg3 RM will be presented in this article. Authenticity certification of the RMs encompasses general characteristics in terms of appearance and solubility; identity in terms of IR, ${ }^{1} \mathrm{H}$ NMR, ${ }^{13} \mathrm{C}$ NMR, mass, and UV spectra analyses; purity in terms of elementary analysis, three different HPLC methods, optical rotation, water content, melting point, residual organic solvents, and TLC; and content in terms of three different HPLC methods. Authenticity document for ginsenosides Rc, Rd, Re, Rf, (20S, 20R)-Rg2, (20E)ginsenoside $\mathrm{F} 4$, and compound $\mathrm{K}$ and establishment of validated analytical procedure for those ginsenosides will be presented elsewhere.

\section{MATERIALS AND METHODS}

\section{Chemicals}

Methanol, acetonitrile, and isopropyl alcohol were HPLC grade and purchased from Burdick \& Jackson (Honeywell International Inc., Muskegon, MI, USA). All other chemicals and solvents were of analytical grade, unless otherwise mentioned. TLC plates were purchased from Merck (HPTLC plate with silica gel 60 F254). Reference ginsenoside $\mathrm{Rg} 3$ was purchased from WaKo (Pure Chemical Industries, Osaka, Japan) for comparisons of purity and identity. 


\section{Isolation and purification of ginsenosides}

Korean red ginseng extract, a water extract obtained from steamed and dried roots of $P$. ginseng, was employed for the isolation and purification of individual ginsenosides. Red ginseng water extract $(1 \mathrm{~kg})$ was dissolved in water to make approximately $20 \%$ to $25 \%$ (w/v) solution. The solution was passed through a Diaion HP20 adsorption column $(8 \mathrm{~L})$, and the resin was washed with 4 wet resin volumes of $20 \%$ ethanol. Crude saponin adsorbed to the macro-reticular resin was eluted with 4 wet resin volumes of pure methanol. The methanol elute was then dried with silica gel powder in vacuo to obtain a pale brown powder. The powder was loaded on a glass column $(15 \times 100 \mathrm{~cm})$ containing $3.5 \mathrm{~L}$ of silica gel and partitioned with solvent mixtures of $\mathrm{CHCl}_{3}-\mathrm{MeOH}-\mathrm{H}_{2} \mathrm{O}$ (12:3:1, 9:3:1, 7:3:1, 4:3:1; lower phase, 4 bed volumes for each mixture). Fractions containing the ginsenosides $\mathrm{Rg} 3$ were dried in vacuo and recrystallized in water until a white powder was formed. The white crystalline amorphous powder was then subjected to preparative HPLC ( $20 \times 250 \mathrm{~mm}$, ODS YMC-Pack, $203 \mathrm{~nm}$ ) with aqueous $45 \% \mathrm{CH}_{3} \mathrm{CN}$ as the mobile phase to purify single ginsenoside. The peak corresponding to each ginsenoside was collected and evaporated to dryness and further dried in a vacuum drier for $5 \mathrm{~d}$. The resultant white powder was stored at $-20^{\circ} \mathrm{C}$ until further use.

\section{Determination of physico-chemical properties of isolated ginsenosides}

Purified (20S)- and (20R)-ginsenoside Rg3 were subjected to authenticity tests to qualify the standard reference material (SRM) for quality control as suggested by Gaedcke et al. [6]. Authenticity of each ginsenosides was certified by determining general characteristics in terms of color, appearance, and solubility. The identities of the ginsenosides were certified by analyzing ${ }^{1} \mathrm{H} \mathrm{NMR},{ }^{13} \mathrm{C}$ NMR, IR, FAB/MS, and UV spectra. The identities of the compounds were verified by $2 \mathrm{D}$ NMR: ${ }^{1} \mathrm{H}-{ }^{13} \mathrm{C}$ correlation spectroscopy (COSY), heteronuclear multiple spectroscopy (HMQC), heteronuclear multiple bond correlation (HMBC), and nuclear overhauser spectroscopy (NOSEY). The purity of the compounds was determined by measuring melting point, elemental composition, water content, residual solvent content, and TLC and HPLC fingerprints obtained by 3 different systems. The quantitation of these compounds was achieved by three different HPLC methods. The water content of ginsenosides was determined on a micro-scale by a Karl Fisher Titrator in accordance with the European pharmacopoeia method [7]. Residual solvent was analyzed by GC/MS after preparing the sample by the headspace method. A UV spectrum was measured at a sample concentration of $0.5 \mathrm{mg} / \mathrm{mL}$ methanol. A $2 \mathrm{mg}$ sample was introduced into the element analyzer. Optical rotation was determined with 5 replicates. Net mass balance of the ginsenosides was calculated as follows: [100\%-water content (\%)-residual solvent content $(\%)] \times$ HPLC purity $(\%) / 100$. Finally, all the physico-chemical property data were compared with the values described in the literature.

\section{Analytical instruments}

NMR (JEOL Ltd., Tokyo, Japan; $500 \mathrm{MHz}$, TMS as the external reference, $20 \mathrm{mg} / \mathrm{mL}$ DMSO- $\left.d_{6}\right), \mathrm{FAB} / \mathrm{MS}$ (JMS-700 Mstation, JEOL Ltd.), IR (Mattson model Galaxy 7020A, Ge-coated $\mathrm{KBr}$ pellet, $3.0 \mathrm{mg} / \mathrm{g}$ ), and UV (V530; Jasco, Tokyo, Japan) spectrometers were used for determining identity. Melting point analyzer (Manstead/ Electrothermal IA9100; Barnstead, Boston, MA, USA), element analyzer (Flash EA1112 series; CE Elantec, Lakewood, NJ, USA), Karl Fisher titrator (831KF; Metrohm, Hartmannsdorf, Germany), and polarimeter (Jasco P-1020, Jasco; filter $589 \mathrm{~mm}$, cylindrical glass cell: $10 \times 100 \mathrm{~mm}$; c, 1.0 in $\mathrm{MeOH}$ ) were used for the determination of purity. Two HPLC systems were employed to determine purity and content: 1) Hewlett-Packard (Wilmington, DE, USA) equipped with a G1313A automatic sample injector, a vacuum degasser unit, a G1311A quatpump, and a G1315A photo diode array detector; 2) Waters (Taunton, MA, USA) equipped with a 2707 automatic sample injector, 1525 binary HPLC pump, and an Alltech Model ELSD 2000 detector.

\section{Preparation of standard solution}

Five milligram of each (20S, 20R)-ginsenoside Rg3 were dissolved in $10 \mathrm{~mL}$ of HPLC grade methanol and diluted until the final concentration was $62.5 \mu \mathrm{g} / \mathrm{mL}$. The diluted standard solution was kept at $-20^{\circ} \mathrm{C}$ until use and stood at room temperature for at least $30 \mathrm{~min}$ prior to use.

\section{Conditions for HPLC analysis}

HPLC analysis of ginsenosides for content determination was performed in 3 different conditions: 1) Discovery HS C18 column $(4.6 \times 250 \mathrm{~mm}, 5 \mu \mathrm{m}$; Supelco, Bellefonte, PA, USA) at room temperature with a sample injection volume of $20 \mu \mathrm{L}$, mobile phase consisted of acetonitrile (solvent A) and water (solvent B), the following program was employed: equilibrated with $\mathrm{A}: \mathrm{B}$ (20:80) for $20 \mathrm{~min}$, linear gradient ranged from A:B $(20: 80)$ to $(35: 65)$ from 20 to $40 \mathrm{~min}, \mathrm{~A}: \mathrm{B}(35: 65)$ to (45:55) from 40 to $52 \mathrm{~min}, \mathrm{~A}: \mathrm{B}(45: 55)$ to $(70: 30)$ from 
52 to $62 \mathrm{~min}, \mathrm{~A}: \mathrm{B}(70: 30)$ to $(100: 0)$ from 62 to $80 \mathrm{~min}$ and finally equilibrated with $A: B(20: 80)$ from 80 to $90 \mathrm{~min}$. The flow rate of the mobile phase was $1.6 \mathrm{~mL} /$ $\mathrm{min}$, and the detector wavelength was set to $203 \mathrm{~nm} ; 2$ ) Symmetry C18 column $(250 \times 4.6 \mathrm{~mm}, 5 \mu \mathrm{m}$; Waters $)$ at room temperature with a sample injection volume of 20 $\mu \mathrm{L}$, the mobile phase consisted of acetonitrile containing $0.1 \%$ formic acid (solvent $\mathrm{A}$ ) and water containing $0.1 \%$ formic acid (solvent $\mathrm{B}$ ). The following program was employed: equilibrated with A:B (20:80) for $20 \mathrm{~min}$, linear gradient started with $\mathrm{A}: \mathrm{B}(20: 80)$ to $(35: 65)$ from 20 to $40 \mathrm{~min}, \mathrm{~A}: \mathrm{B}(35: 65)$ to $(45: 55)$ from 40 to $52 \mathrm{~min}$, $\mathrm{A}: \mathrm{B}(45: 55)$ to (70:30) from 52 to $62 \mathrm{~min}, \mathrm{~A}: \mathrm{B}(70: 30)$ to (100:0) from 62 to $80 \mathrm{~min}$ and finally equilibrated with A:B (20:80) from 80 to $90 \mathrm{~min}$. The flow rate of the mobile phase was $1.6 \mathrm{~mL} / \mathrm{min}$, and the detector wavelength was set to $203 \mathrm{~nm}$; 3) Prevail carbohydrate ES column $(250 \times 4.6 \mathrm{~mm}, 5 \mu \mathrm{m}$; Alltech, Deerfield, IL, USA) at room temperature with a sample injection volume of $10 \mu \mathrm{L}$, the mobile phase consisted of acetonitrile/ $\mathrm{H}_{2} \mathrm{O} /$ isopropyl alcohol=80/5/15 (solvent $\mathrm{A}$ ) and acetonitrile/ $\mathrm{H}_{2} \mathrm{O} /$ isopropyl alcohol=80/15/15 (solvent B), the following program was employed: linear gradient ranged from $\mathrm{A}: \mathrm{B}(75: 25)$ to $(15: 85)$ for $28 \mathrm{~min}, \mathrm{~A}: \mathrm{B}(15: 85)$ to $(20: 80)$ from 28 to $35 \mathrm{~min}, \mathrm{~A}: \mathrm{B}(20: 80)$ to $(25: 75)$ from 35 to $45 \mathrm{~min}, \mathrm{~A}: \mathrm{B}(25: 75)$ to $(10: 90)$ from 45 to $50 \mathrm{~min}, \mathrm{~A}: \mathrm{B}$ $(10: 90)$ to $(0: 100)$ from 50 to $55 \mathrm{~min}$ and finally equilibrated with A:B (75:25) from 55 to $60 \mathrm{~min}$. The flow rate of the mobile phase was $1.0 \mathrm{~mL} / \mathrm{min}$, and the detector was ELSD (gain 8, gas flow rate 2.0 SLPM drift tube temperature $90^{\circ} \mathrm{C}$ ).

\section{Statistical analyses}

Data were presented as the means \pm SEM. Statistical analyses were performed using Student's $t$-test in Excel (Microsoft, Redmont, WA, USA). Differences were considered significant when $p<0.05$.

\section{RESULTS}

\section{Isolation and purification of individual ginsen- osides}

We could isolate ten grams of (20S)- and (20R)ginsenoside $\mathrm{Rg} 3$ via adsorption chromatography, silica gel partition chromatography, recrystallization, and preparative HPLC fractionation. Fractions obtained from $\mathrm{CHCl}_{3}-\mathrm{MeOH}-\mathrm{H}_{2} \mathrm{O}$ (12:3:1) gave predominantly (20E)ginsenoside $\mathrm{F} 4$ and that of $\mathrm{CHCl}_{3}-\mathrm{MeOH}-\mathrm{H}_{2} \mathrm{O}(9: 3: 1)$ afforded mainly ginsenoside $\mathrm{Rg} 3$. The recrystallized white powder was purified by preparative HPLC $(20 \times 250 \mathrm{~mm}$, ODS YMC-Pack, $203 \mathrm{~nm}$ ) with $45 \% \mathrm{CH}_{3} \mathrm{CN}$. A peak corresponding to (20S)- and (20R)-ginsenoside Rg3 was collected, dried in vacuo, and further dried in a vacuum drier for $5 \mathrm{~d}$ to obtain a white amorphous powder.

\section{General characteristics of the isolated (20S)- and (20R)-ginsenoside $\mathbf{R g} 3$}

Isolated (20S)- and (20R)-ginsenoside Rg3 were subjected to various physico-chemical analyses, including general characteristics. As shown in Table 1, (20S)- and (20R)-ginsenoside Rg3 exhibited as white amorphous powders. (20S)-ginsenoside Rg3 was readily soluble in cold $\mathrm{H}_{2} \mathrm{O}, \mathrm{EtOH}, \mathrm{MeOH}$, and $\mathrm{CH}_{3} \mathrm{CN}$, whereas $(20 R)$ ginsenoside $\mathrm{Rg} 3$ was readily soluble in DMSO and sparingly soluble in $\mathrm{H}_{2} \mathrm{O}$ and $\mathrm{CH}_{3} \mathrm{CN}$.

\section{Identification of the isolated (20S)- and (20R)- ginsenoside Rg3}

Isolated (20S)- and (20R)-ginsenoside $\mathrm{Rg} 3$ were subjected to various spectrometric analyses for identification. Interpretation of ${ }^{1} \mathrm{H}$ and ${ }^{13} \mathrm{C}$ NMR spectra together with ${ }^{1} \mathrm{H}-{ }^{1} \mathrm{H}$ COSY, HMQC, HMBC, and NOESY spectra allowed us to assign all the ${ }^{1} \mathrm{H}$ and ${ }^{13} \mathrm{C}$ NMR signals (Table 2). Determining the chemical shift (ppm), multiplicity and coupling constant of each proton signal was also achieved by interpretation of NMR spectra. Comparing

Table 1. General characteristics of isolated (20S)- and (20R)-ginsenoside Rg3

\begin{tabular}{lll}
\hline & \multicolumn{1}{c}{$(20 S)$-ginsenoside $\mathrm{Rg} 3$} & $(20 R)$-ginsenoside $\mathrm{Rg} 3$ \\
\hline Biological source & Panax ginseng Meyer & P. ginseng Meyer \\
CAS registry no. & $14197-60-5$ & $38243-03-7$ \\
Empirical formula & $\mathrm{C}_{42} \mathrm{H}_{72} \mathrm{O}_{13}$ & $\mathrm{C}_{42} \mathrm{H}_{72} \mathrm{O}_{13}$ \\
Molar mass & $785.023 \mathrm{~g} / \mathrm{molar}$ & $785.023 \mathrm{~g} / \mathrm{molar}$ \\
Accurate mass & $784.497 \mathrm{~g} / \mathrm{molar}$ & $784.497 \mathrm{~g} / \mathrm{molar}$ \\
Appearance & White amorphous powder, solid at room temperature & White amorphous powder, solid at room temperature \\
Solubility $\left(25^{\circ} \mathrm{C}\right)$ & Water, anhydrous ethanol and methanol & Sparingly soluble in water, anhydrous ethanol and methanol
\end{tabular}

CAS, Chemical Abstracts Service. 
Table 2. NMR spectra assignment for (20S)- and (20R)-ginsenoside Rg3

\begin{tabular}{|c|c|c|c|c|c|c|}
\hline \multirow{2}{*}{$\begin{array}{l}\text { Proton } \\
\text { at } \mathrm{C} \text { atom }\end{array}$} & \multicolumn{3}{|c|}{ (20S)-ginsenoside Rg3 } & \multicolumn{3}{|c|}{$(20 R)$-ginsenoside Rg3 } \\
\hline & $\begin{array}{l}\text { Chemical } \\
\text { shift (ppm) }\end{array}$ & $\begin{array}{l}\text { Multiplicity, coupling } \\
\text { constants }(\mathrm{Hz})\end{array}$ & $\begin{array}{l}\text { Reference chemical } \\
\text { shift (ppm) }[8]\end{array}$ & $\begin{array}{l}\text { Chemical } \\
\text { shift (ppm) }\end{array}$ & $\begin{array}{l}\text { Multiplicity, coupling } \\
\text { constants }(\mathrm{Hz})\end{array}$ & $\begin{array}{l}\text { Reference chemical } \\
\text { shift (ppm) [9] }\end{array}$ \\
\hline \multirow[b]{2}{*}{1} & 1.54 & $\mathrm{~m} ; 1 \mathrm{H}$ & 1.55 & 1.56 & $\mathrm{~m} ; 1 \mathrm{H}$ & 1.54 \\
\hline & 0.94 & $\mathrm{~m} ; 1 \mathrm{H}$ & 0.93 & 0.94 & $\mathrm{~m} ; 1 \mathrm{H}$ & 0.92 \\
\hline \multirow{2}{*}{2} & 1.87 & $\mathrm{~m} ; 1 \mathrm{H}$ & 1.87 & 1.87 & $\mathrm{~m} ; 1 \mathrm{H}$ & 1.86 \\
\hline & 1.55 & $\mathrm{~m} ; 1 \mathrm{H}$ & 1.55 & 1.57 & $\mathrm{~m} ; 1 \mathrm{H}$ & 1.55 \\
\hline 3 & 3.02 & $\mathrm{~m} ; 1 \mathrm{H}$ & 3.01 & 3.02 & $\mathrm{~m} ; 1 \mathrm{H}$ & 3.00 \\
\hline 5 & 0.69 & $\mathrm{~d}(\mathrm{~J}=11.5 \mathrm{~Hz}) ; 1 \mathrm{H}$ & 0.69 & 0.70 & $\mathrm{~d}(\mathrm{~J}=11.5 \mathrm{~Hz}) ; 1 \mathrm{H}$ & 0.69 \\
\hline \multirow{2}{*}{6} & 1.46 & $\mathrm{~m} ; 1 \mathrm{H}$ & 1.46 & 1.46 & $\mathrm{~m} ; 1 \mathrm{H}$ & 1.46 \\
\hline & 1.36 & $\mathrm{~m} ; 1 \mathrm{H}$ & 1.36 & 1.36 & $\mathrm{~m} ; 1 \mathrm{H}$ & 1.36 \\
\hline \multirow{2}{*}{7} & 1.44 & $\mathrm{~m} ; 1 \mathrm{H}$ & 1.43 & 1.44 & $\mathrm{~m} ; 1 \mathrm{H}$ & 1.44 \\
\hline & 1.17 & $\mathrm{~m} ; 1 \mathrm{H}$ & 1.19 & 1.17 & $\mathrm{~m} ; 1 \mathrm{H}$ & 1.16 \\
\hline 9 & 1.33 & $\mathrm{~m} ; 1 \mathrm{H}$ & 1.34 & 1.35 & $\mathrm{~m} ; 1 \mathrm{H}$ & 1.34 \\
\hline \multirow{2}{*}{11} & 1.69 & $\mathrm{~m} ; 1 \mathrm{H}$ & 1.67 & 1.70 & $\mathrm{~m} ; 1 \mathrm{H}$ & 1.67 \\
\hline & 1.10 & $\mathrm{~m} ; 1 \mathrm{H}$ & 1.11 & 1.13 & $\mathrm{~m} ; 1 \mathrm{H}$ & 1.11 \\
\hline 12 & 3.37 & $\mathrm{~m} ; 1 \mathrm{H}$ & 3.37 & 3.37 & $\mathrm{~m} ; 1 \mathrm{H}$ & 3.37 \\
\hline $12-\mathrm{OH}$ & 6.05 & $\mathrm{~s} ; 1 \mathrm{H}$ & & 6.12 & $\mathrm{~S} ; 1 \mathrm{H}$ & 5.81 \\
\hline 13 & 1.57 & $\mathrm{~m} ; 1 \mathrm{H}$ & 1.56 & 1.58 & $\mathrm{~m} ; 1 \mathrm{H}$ & 1.56 \\
\hline \multirow{2}{*}{15} & 1.41 & $\mathrm{~m} ; 1 \mathrm{H}$ & 1.41 & 1.41 & $\mathrm{~m} ; 1 \mathrm{H}$ & 1.40 \\
\hline & 0.93 & $\mathrm{~m} ; 1 \mathrm{H}$ & 0.93 & 0.93 & $\mathrm{~m} ; 1 \mathrm{H}$ & 0.93 \\
\hline \multirow{2}{*}{16} & 1.72 & $\mathrm{~m} ; 1 \mathrm{H}$ & 1.73 & 1.72 & $\mathrm{~m} ; 1 \mathrm{H}$ & 1.71 \\
\hline & 1.21 & $\mathrm{~m} ; 1 \mathrm{H}$ & 1.21 & 1.21 & $\mathrm{~m} ; 1 \mathrm{H}$ & 1.20 \\
\hline 17 & 1.89 & $\mathrm{~m} ; 1 \mathrm{H}$ & 1.89 & 1.89 & $\mathrm{~m} ; 1 \mathrm{H}$ & 1.88 \\
\hline 18 & 0.91 & $\mathrm{~s} ; 3 \mathrm{H}$ & 0.89 & 0.92 & $\mathrm{~s} ; 3 \mathrm{H}$ & 0.89 \\
\hline 19 & 0.82 & $\mathrm{~s} ; 3 \mathrm{H}$ & 0.80 & 0.82 & $\mathrm{~s} ; 3 \mathrm{H}$ & 0.80 \\
\hline $20-\mathrm{OH}$ & 5.92 & $\mathrm{~s} ; 1 \mathrm{H}$ & & 5.95 & $\mathrm{~s} ; 1 \mathrm{H}$ & 5.74 \\
\hline 21 & 1.05 & $\mathrm{~s} ; 3 \mathrm{H}$ & 1.01 & 1.00 & $\mathrm{~s} ; 3 \mathrm{H}$ & 0.96 \\
\hline \multirow{2}{*}{22} & 1.38 & $\mathrm{~m} ; 1 \mathrm{H}$ & 1.39 & 1.35 & $\mathrm{~m} ; 1 \mathrm{H}$ & 1.30 \\
\hline & 1.28 & $\mathrm{~m} ; 1 \mathrm{H}$ & 1.28 & 1.35 & $\mathrm{~m} ; 1 \mathrm{H}$ & 1.30 \\
\hline \multirow{2}{*}{23} & 2.07 & $\mathrm{~m} ; 1 \mathrm{H}$ & 2.07 & 2.01 & $\mathrm{~m} ; 1 \mathrm{H}$ & 1.99 \\
\hline & 1.90 & $\mathrm{~m} ; 1 \mathrm{H}$ & 1.90 & 2.01 & $\mathrm{~m} ; 1 \mathrm{H}$ & 1.99 \\
\hline 24 & 5.09 & $\mathrm{~m} ; 1 \mathrm{H}$ & 5.09 & 5.08 & $\mathrm{~m} ; 1 \mathrm{H}$ & 5.05 \\
\hline 26 & 1.65 & $\mathrm{~s} ; 3 \mathrm{H}$ & 1.63 & 1.63 & $\mathrm{~s} ; 3 \mathrm{H}$ & 1.61 \\
\hline 27 & 1.57 & $\mathrm{~s} ; 3 \mathrm{H}$ & 1.55 & 1.57 & $\mathrm{~s} ; 3 \mathrm{H}$ & 1.54 \\
\hline 28 & 0.98 & $\mathrm{~s} ; 3 \mathrm{H}$ & 0.97 & 0.98 & $\mathrm{~s} ; 3 \mathrm{H}$ & 0.96 \\
\hline 29 & 0.75 & $\mathrm{~s} ; 3 \mathrm{H}$ & 0.73 & 0.75 & $\mathrm{~s} ; 3 \mathrm{H}$ & 0.72 \\
\hline 30 & 0.82 & $\mathrm{~s} ; 3 \mathrm{H}$ & 0.81 & 0.82 & $\mathrm{~s} ; 3 \mathrm{H}$ & 0.81 \\
\hline
\end{tabular}


Kim et al. Documentation of (20S)- and (20R)-ginsenoside Rg3 as standard reference materials

Table 2. (Continued)

\begin{tabular}{|c|c|c|c|c|c|c|}
\hline \multirow{2}{*}{$\begin{array}{l}\text { Proton } \\
\text { at } \mathrm{C} \text { atom }\end{array}$} & \multicolumn{3}{|c|}{ (20S)-ginsenoside $\operatorname{Rg} 3$} & \multicolumn{3}{|c|}{ (20R)-ginsenoside $\operatorname{Rg} 3$} \\
\hline & $\begin{array}{l}\text { Chemical } \\
\text { shift (ppm) }\end{array}$ & $\begin{array}{l}\text { Multiplicity, coupling } \\
\text { constants }(\mathrm{Hz})\end{array}$ & $\begin{array}{l}\text { Reference chemical } \\
\text { shift (ppm) [8] }\end{array}$ & $\begin{array}{l}\text { Chemical } \\
\text { shift (ppm) }\end{array}$ & $\begin{array}{l}\text { Multiplicity, coupling } \\
\text { constants }(\mathrm{Hz})\end{array}$ & $\begin{array}{l}\text { Reference chemical } \\
\text { shift (ppm) [9] }\end{array}$ \\
\hline \multicolumn{7}{|c|}{ Glucose 1 at $\mathrm{C} 3$} \\
\hline 1 & 4.29 & $\mathrm{~d}(\mathrm{~J}=7.0 \mathrm{~Hz}) ; 1 \mathrm{H}$ & 4.26 & 4.29 & $\mathrm{~d}(\mathrm{~J}=7.0 \mathrm{~Hz}) ; 1 \mathrm{H}$ & 4.24 \\
\hline 2 & 3.37 & $\mathrm{~m} ; 1 \mathrm{H}$ & 3.31 & 3.38 & $\mathrm{~m} ; 1 \mathrm{H}$ & 3.28 \\
\hline 3 & 3.38 & $\mathrm{~m} ; 1 \mathrm{H}$ & 3.34 & 3.39 & $\mathrm{~m} ; 1 \mathrm{H}$ & 3.33 \\
\hline & 5.78 & $\mathrm{~d} ; 1 \mathrm{H}$ & & 5.77 & $\mathrm{~d} ; 1 \mathrm{H}$ & 5.67 \\
\hline \multirow[t]{2}{*}{4} & 3.08 & $\mathrm{~m} ; 1 \mathrm{H}$ & 3.07 & 3.08 & $\mathrm{~m} ; 1 \mathrm{H}$ & 3.06 \\
\hline & 5.22 & $\mathrm{~d} ; 1 \mathrm{H}$ & & 5.19 & $\mathrm{~d} ; 1 \mathrm{H}$ & 4.98 \\
\hline 5 & 3.08 & $\mathrm{~m} ; 1 \mathrm{H}$ & 3.07 & 3.08 & $\mathrm{~m} ; 1 \mathrm{H}$ & 3.06 \\
\hline 6 & 3.64 & $\mathrm{~m} ; 1 \mathrm{H}$ & 3.64 & 3.67 & $\mathrm{~m} ; 1 \mathrm{H}$ & 3.63 \\
\hline \multirow{2}{*}{$6-\mathrm{OH}$} & 3.41 & $\mathrm{~m} ; 1 \mathrm{H}$ & 3.41 & 3.47 & $\mathrm{~m} ; 1 \mathrm{H}$ & 3.40 \\
\hline & 4.71 & $\mathrm{t} ; 1 \mathrm{H}$ & & 4.67 & $\mathrm{t} ; 1 \mathrm{H}$ & 4.37 \\
\hline \multicolumn{7}{|c|}{ Glucose 2 at $\mathrm{C} 3$} \\
\hline 1 & 4.48 & $\mathrm{~d}(\mathrm{~J}=7.5 \mathrm{~Hz}) ; 1 \mathrm{H}$ & 4.42 & 4.48 & $\mathrm{~d}(\mathrm{~J}=7.5 \mathrm{~Hz}) ; 1 \mathrm{H}$ & 4.40 \\
\hline 2 & 3.00 & $\mathrm{~m} ; 1 \mathrm{H}$ & 2.98 & 3.02 & $\mathrm{~m} ; 1 \mathrm{H}$ & 2.97 \\
\hline 3 & 5.54 & $\mathrm{~d} ; 1 \mathrm{H}$ & & 5.52 & $\mathrm{~d} ; 1 \mathrm{H}$ & 5.40 \\
\hline $3-\mathrm{OH}$ & 3.12 & $\mathrm{~m} ; 1 \mathrm{H}$ & 3.12 & 3.12 & $\mathrm{~m} ; 1 \mathrm{H}$ & 3.11 \\
\hline 4 & 5.11 & Overlapped with other signals & & 5.08 & Overlapped & 4.90 \\
\hline $4-\mathrm{OH}$ & 3.11 & $\mathrm{~m} ; 1 \mathrm{H}$ & 3.11 & 3.12 & $\mathrm{~m} ; 1 \mathrm{H}$ & 3.10 \\
\hline 5 & 5.11 & Overlapped with other signals & & 5.08 & Overlapped & 4.83 \\
\hline 6 & 3.02 & $\mathrm{~m} ; 1 \mathrm{H}$ & 3.02 & 3.03 & $\mathrm{~m} ; 1 \mathrm{H}$ & 3.01 \\
\hline \multirow{3}{*}{$6-\mathrm{OH}$} & 3.64 & $\mathrm{~m} ; 1 \mathrm{H}$ & 3.60 & 3.66 & $\mathrm{~m} ; 1 \mathrm{H}$ & 3.58 \\
\hline & 3.47 & $\mathrm{~m} ; 1 \mathrm{H}$ & 3.47 & 3.47 & $\mathrm{~m} ; 1 \mathrm{H}$ & 3.46 \\
\hline & 4.61 & $\mathrm{t} ; 1 \mathrm{H}$ & & 4.53 & $\mathrm{t} ; 1 \mathrm{H}$ & 4.10 \\
\hline
\end{tabular}

\begin{tabular}{|c|c|c|c|c|}
\hline \multirow{2}{*}{$\mathrm{C}$ atom } & \multicolumn{2}{|c|}{ (20S)-ginsenoside Rg3 (ppm) } & \multicolumn{2}{|c|}{$(20 R)$-ginsenoside Rg3 (ppm) } \\
\hline & Chemical shift & Reference chemical shift [8] & Chemical shift & Reference chemical shift [9] \\
\hline 1 & 38.5 (overlapped with solvent) & 38.5 & Overlapped with solvent (49.0) & 38.4 \\
\hline 2 & 25.6 & 25.7 & 25.5 & 25.8 \\
\hline 3 & 88.4 & 88.1 & 88.4 & 88.0 \\
\hline 4 & 38.7 (overlapped with solvent) & 38.6 & Overlapped with solvent (49.0) & 38.2 \\
\hline 5 & 55.4 & 55.4 & 55.5 & 55.4 \\
\hline 6 & 17.6 & 17.6 & 17.6 & 17.7 \\
\hline 7 & 34.2 & 34.3 & 34.3 & 34.3 \\
\hline 8 & 39 (overlapped with solvent) & 36.2 & Overlapped with solvent (49.0) & 36.2 \\
\hline 9 & 49.3 & 49.3 & 49.1 & 49.3 \\
\hline 10 & 36.1 & 38.8 & 36.1 & 38.6 \\
\hline 11 & 30.7 & 30.8 & 30.7 & 31.0 \\
\hline 12 & 69.8 & 69.7 & 69.7 & 69.5 \\
\hline 13 & 47.2 & 47.3 & 47.8 & 47.8 \\
\hline 14 & 50.8 & 50.4 & 50.9 & 50.1 \\
\hline 15 & 30.4 & 30.5 & 30.5 & 30.4 \\
\hline 16 & 25.8 & 25.8 & 25.5 & 25.5 \\
\hline 17 & 53.2 & 53.4 & 49.3 & 49.4 \\
\hline 18 & 15.3 & 15.4 & 15.2 & 16.4 \\
\hline 19 & 15.8 & 15.8 & 15.8 & 15.8 \\
\hline 20 & 72.2 & 71.8 & 72.2 & 71.4 \\
\hline
\end{tabular}


Table 2. (Continued)

\begin{tabular}{|c|c|c|c|c|}
\hline \multirow{2}{*}{$\mathrm{C}$ atom } & \multicolumn{2}{|c|}{ (20S)-ginsenoside Rg3 (ppm) } & \multicolumn{2}{|c|}{$(20 R)$-ginsenoside $\mathrm{Rg} 3(\mathrm{ppm})$} \\
\hline & Chemical shift & Reference chemical shift [8] & Chemical shift & Reference chemical shift [9] \\
\hline 21 & 26.0 & 26.3 & 21.9 & 22.0 \\
\hline 22 & 34.8 & 34.8 & 41.8 & 41.9 \\
\hline 23 & 21.8 & 21.8 & 21.3 & 21.4 \\
\hline 24 & 125.2 & 125.4 & 124.9 & 124.9 \\
\hline 25 & 130.3 & 129.8 & 130.3 & 129.5 \\
\hline 26 & 25.4 & 25.4 & 25.4 & 25.4 \\
\hline 27 & 17.4 & 17.5 & 17.3 & 17.5 \\
\hline 28 & 27.3 & 27.4 & 27.3 & 27.4 \\
\hline 29 & 15.7 & 15.7 & 15.7 & 15.7 \\
\hline 30 & 16.3 & 16.5 & 16.6 & 15.4 \\
\hline \multicolumn{5}{|l|}{ Glucose 1 at $\mathrm{C} 3$} \\
\hline 1 & 103.2 & 103.5 & 103.3 & 103.5 \\
\hline 2 & 80.4 & 81.2 & 80.5 & 81.0 \\
\hline 3 & 76.5 & 76.4 & 76.5 & 76.4 \\
\hline 4 & 69.8 & 69.9 & 69.8 & 69.7 \\
\hline 5 & 76.1 & 76.3 & 76.1 & 76.2 \\
\hline 6 & 60.9 & 60.9 & 60.9 & 60.9 \\
\hline \multicolumn{5}{|l|}{ Glucose 2 at $\mathrm{C} 3$} \\
\hline 1 & 103.4 & 103.8 & 103.4 & 103.8 \\
\hline 2 & 74.9 & 75.2 & 74.9 & 75.0 \\
\hline 3 & 76.0 & 76.0 & 76.0 & 75.7 \\
\hline 4 & 69.8 & 69.8 & 69.8 & 69.6 \\
\hline 5 & 76.7 & 76.7 & 76.7 & 76.7 \\
\hline 6 & 60.9 & 60.7 & 60.9 & 60.7 \\
\hline
\end{tabular}

${ }^{1} \mathrm{H}$ and ${ }^{13} \mathrm{C}$ NMR spectra assignments with data in the literature cited suggest that the reference ginsenosides isolated and analyzed by our laboratory are identical. IR spectra were in accordance with the structure of (20S)and $(20 R)$-ginsenoside $\mathrm{Rg} 3$ and corresponded to the reference spectra. Other IR wavenumbers were not significant to the structure (Table 3). Fragmentation ion of $m /$ $z 807.6$ in positive FAB/MS mode of (20S)- and (20R)ginsenoside $\mathrm{Rg} 3$ revealed $[\mathrm{M}+\mathrm{Na}]^{+}$and that of $\mathrm{m} / \mathrm{z} 783.5$ in negative mode peak indicated $[\mathrm{M}-\mathrm{H}]$. Fragmentation ions of $m / z$ 783.5, 621.4, and 459.3 demonstrated [M-H]', $\left[\mathrm{M}-\mathrm{H}-\mathrm{C}_{6} \mathrm{H}_{10} \mathrm{O}_{5}\right]^{\prime}$, and $\left[\mathrm{M}-\mathrm{H}-\mathrm{C}_{6} \mathrm{H}_{10} \mathrm{O}_{5}-\mathrm{C}_{6} \mathrm{H}_{10} \mathrm{O}_{5}\right]^{\text {, r respec- }}$ tively. The UV spectra of (20S)- and (20R)-ginsenoside Rg3 showed no characteristic absorption patterns. The two compounds demonstrated end absorption with absorption maximum at the vicinity of $203 \mathrm{~nm}$. Identification also took place within the framework of the HPLC (Hewlett Packard) analyses with a photodiode array detector at $203 \mathrm{~nm}$, demonstrating the corresponding UV spectra for those peaks of (20S)- and (20R)-ginsenoside $\mathrm{Rg} 3$. There was absolutely no difference in UV spectra between the two compounds. Based on those spectrometric data, we could identify authenticity and chemical structures of the two ginsenosides (Fig. 1).

\section{Purity of isolated (20S)- and (20R)-ginsenoside Rg3}

Purity was determined by analyzing melting point, elemental composition, and HPLC purity in 3 different conditions, water and residual solvent content, optical rotation, and TLC. The melting points of the (20S)- and (20R)-ginsenoside $\mathrm{Rg} 3$ were determined to be $295.47^{\circ} \mathrm{C}$ and $248.55^{\circ} \mathrm{C}$, respectively (Table 4). These values correspond with the data in the literature [8], indicating a valid temperature range between $293^{\circ} \mathrm{C}$ to $303^{\circ} \mathrm{C}$, which certifies the purity of the (20S)- and (20R)-ginsenoside Rg3. Elemental analysis was tested in triplicate, and the result 
Kim et al. Documentation of (20S)- and (20R)-ginsenoside Rg3 as standard reference materials

Table 3. Spectrophotometric data for the isolated (20S)- and (20R)-ginsenoside Rg3

\begin{tabular}{|c|c|c|c|c|}
\hline \multirow{2}{*}{ Parameter } & \multicolumn{2}{|c|}{ (20S)-ginsenoside $\mathrm{Rg} 3$} & \multicolumn{2}{|c|}{$(20 R)$-ginsenoside $\operatorname{Rg} 3$} \\
\hline & Isolated & Reference [8] & Isolated & Reference [8] \\
\hline IR & $3413,2947,1636,1385,1076$ & $3400,2930,1635,1390,1080$ & $3413,2947,1636,1385,1076$ & $3400,2930,1635,1390,1080$ \\
\hline UV & 203 & 203 & 203 & 203 \\
\hline $\mathrm{FAB} / \mathrm{MS}$ & $\begin{array}{l}{[\mathrm{M}+\mathrm{Na}]^{+} 807.6} \\
{[\mathrm{M}-\mathrm{H}]^{-} 783.8}\end{array}$ & $\begin{array}{l}807.4 \\
783.5\end{array}$ & $\begin{array}{l}{[\mathrm{M}+\mathrm{Na}]^{+} 807.6} \\
{[\mathrm{M}-\mathrm{H}]^{-} 783.8}\end{array}$ & $\begin{array}{l}807.4 \\
783.5\end{array}$ \\
\hline
\end{tabular}

IR, infrared spectroscopy; FAB/MS, fast atom bombardment mass spectrometry.

Table 4. Major Purity data for (20S)- and (20R)-ginsenoside Rg3

\begin{tabular}{|c|c|c|c|c|c|}
\hline & & \multicolumn{2}{|c|}{ (20S)-ginsenoside Rg3 } & \multicolumn{2}{|c|}{$(20 R)$-ginsenoside $\mathrm{Rg} 3$} \\
\hline & & Isolated & Reference & Isolated & Reference \\
\hline Melting point & & $295.47^{\circ} \mathrm{C}$ & $293^{\circ} \mathrm{C}-303^{\circ} \mathrm{C}[8]$ & $248.55^{\circ} \mathrm{C}$ & $236^{\circ} \mathrm{C}-238^{\circ} \mathrm{C}$ \\
\hline Optical rotation & & $\begin{array}{c}+0.382 \\
\text { (c, } 0.9 \text { in DMSO) }\end{array}$ & $+8.5,+13.2[10,11]$ & $\begin{array}{c}-0.88 \\
\text { (c, } 1.0 \text { in DMSO) }\end{array}$ & $+8.5[12,13]$ \\
\hline Water content & & \multicolumn{2}{|c|}{$0.534 \%$} & \multicolumn{2}{|c|}{$0.920 \%$} \\
\hline $\operatorname{TLC}\left(\mathrm{R}_{\mathrm{f}}\right)$ & & \multicolumn{2}{|c|}{$0.48^{1)}$} & \multicolumn{2}{|c|}{$0.48^{1)}$} \\
\hline \multirow[t]{3}{*}{ HPLC (rt min) } & Method 1 & \multicolumn{2}{|c|}{55.620} & \multicolumn{2}{|c|}{56.812} \\
\hline & Method 2 & \multicolumn{2}{|c|}{59.603} & \multicolumn{2}{|c|}{60.449} \\
\hline & Method 3 & \multicolumn{2}{|c|}{18.920} & \multicolumn{2}{|c|}{18.920} \\
\hline
\end{tabular}

rt, retention time.

1) Silica gel $60 \mathrm{~F}_{254}, \mathrm{CHCl}_{3}: \mathrm{MeOH}: \mathrm{H}_{2} \mathrm{O} / 9: 3: 1$, lower phase.
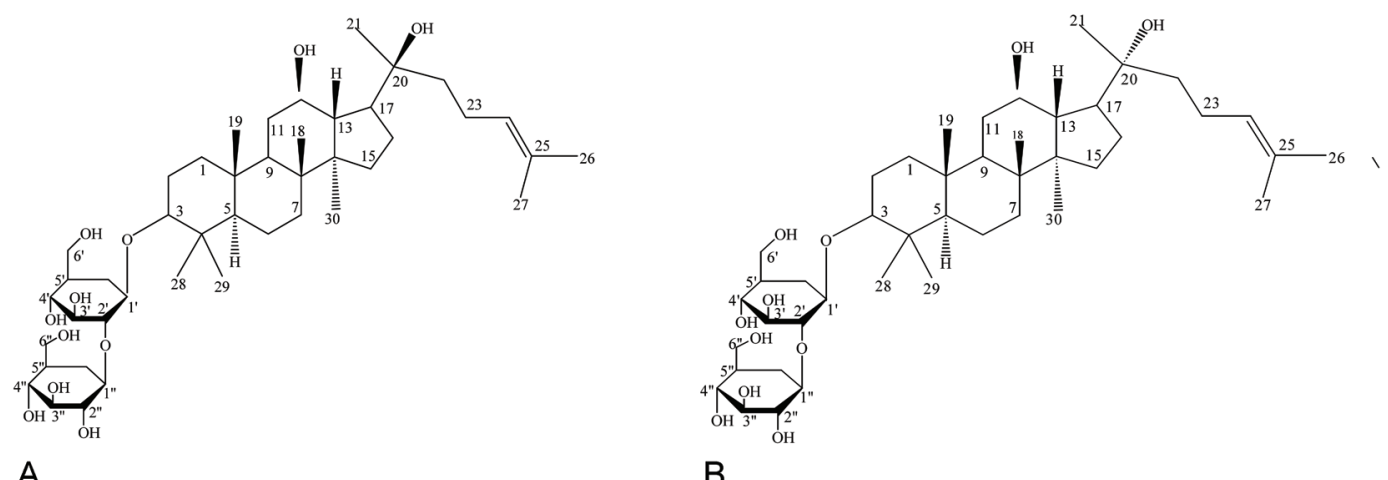

Fig. 1. Chemical structures of (20S)-ginsenosides Rg3 (A) and (20R)-ginsenosides Rg3 (B).

was expressed as a mean value. The elemental composition of (20S)- and (20R)-ginsenoside $\operatorname{Rg} 3$ was $63.28 \%$ carbon, $9.64 \%$ hydrogen, and $24.31 \%$ oxygen. These values coincided with the theoretical values: $\mathrm{C}_{42} \mathrm{H}_{72} \mathrm{O}_{13}$; $42 \times 12.011(64.26 \%)+72 \times 1.0079(9.24 \%)+13 \times 15.999$ (24.31\%).

The optical rotation of the isolated (20S)-ginsenoside Rg3 was +0.382 (c, 0.9 in DMSO), and there was a slight difference between the value obtained with our isolate and the reference value. The optical rotation of the isolated (20R)-ginsenoside Rg3 was -0.88 (c, 0.9 in DMSO), and there was a slight difference between the value ob- tained with our isolate and the reference value (Table 4).

The 1st HPLC (reverse phase, aqueous $\mathrm{CH}_{3} \mathrm{CN}$ ) fingerprint for the (20S)-ginsenoside $\mathrm{Rg} 3$ showed no peak derived from other organic contaminants or ginsenosides except the peak of (20S)-ginsenoside Rg3. The peak purity was also determined by means of spectral analysis. The calculated peak purity value (purity angle, 10.070; $0.400)$ was much lower than the threshold (16.412, $0.457)$, which means that the peak can be regarded as being spectrally homogeneous. The 2nd HPLC (normal phase, aqueous $\mathrm{CH}_{3} \mathrm{CN}$ ) fingerprint demonstrated the same result as that in the first HPLC condition. The peak 
purity was also determined by means of spectral analysis. The calculated peak purity value (purity angle, 10.070; $0.400)$ is lower than the threshold $(16.412,0.457)$, which means that the peak could be regarded as being spectrally homogeneous. The 3rd HPLC fingerprint for ginsenoside $\mathrm{Rg} 3$ was exactly same as that in the 1 st and 2nd. In addition, the three HPLC fingerprints for ginsenoside Rg3 also demonstrated the same purity properties (Table 4). Residual organic solvents, such as $\mathrm{CHCl}_{3}, \mathrm{MeOH}$ and $\mathrm{BuOH}$ used in the process of isolation and purification, were not detected by gas chromatography. The water content of (20S)- and (20R)-ginsenoside Rg3 reference material was determined on a micro-scale by means of a Karl Fisher titration in accordance with the method in European pharmacopoeia. The water content of (20S)and (20R)-ginsenoside $\operatorname{Rg} 3$ equaled $0.534 \%$ and $0.920 \%$, respectively. On a normal phase silica gel TLC plate, the Rf value for (20S)- and (20R)-ginsenoside Rg3 reference material accounted for 0.48 in the given chromatographic conditions. No other band attributed to contamination of other ginsenosides was detected. The small peaks at approximately $5 \mathrm{~min}$ were derived from the methanol solvent used to dissolve the ginsenosides.

\section{Content quantification of isolated (20S)- and (20R)- ginsenoside Rg3}

Content of isolated (20S)- and (20R)-ginsenoside Rg3 accounted for $100 \%$ when measured solely by three different HPLC systems. When the same amount of those ginsenosides was injected 6 times to determine reproducibility, HPLC method 1 exhibited the smallest deviation in peak area.

\section{Mass balance of the isolated (20S)- and (20R)- ginsenoside $\mathbf{R g} \mathbf{3}$}

Mass balance could be calculated after obtaining the water content, content of residual solvent, and HPLC purity in three different systems. Residual solvent was not detected by GC/MS in those two isolated ginsenosides. The HPLC purity of those two compounds was $100 \%$. Therefore, the only impurity we have to take into account when calculating net mass balance was water. The water contents of (20S)- and (20R)-ginsenoside Rg3 were $0.534 \%$ and $0.920 \%$, respectively. Taking these data together, the mass balance for (20S)- and (20R)-ginsenoside $\operatorname{Rg} 3$ amounted to $99.466 \%$ and $99.080 \%$, respectively.

\section{DISCUSSION}

As previously mentioned, ginseng is classified either as a medicine or health food supplement depending on the country. Therefore, quality control should be GMPbased, which requires $\mathrm{ICH}$-guidelines conforming to validated analytical procedures in those countries in which ginseng is categorized as a medicine. In addition, validated quantitative analytical procedures should be constructed before initiating pharmacodynamic and clinical studies to certify batch analysis data for the employed samples. Otherwise, the pharmacodynamic and clinical results will not be accepted by the competent authority of the relevant government. Furthermore, validated analytical procedures cannot be fulfilled without the standard reference materials. In a majority of countries, including EU and Korea, ginsenoside Rb1 and Rg1 are accepted as the reference materials for ginseng preparations. In contrast, China requires additional quantitative analysis of Re, and the EU requires qualitative analysis of Rf. In cases of qualitative analysis, a high-purity standard reference material is not required. However, high purity standard reference materials of ginsenoside Rb1 and Rg1 are essential for the registration of ginseng products as a medicine in the EU. Currently, the analytical method for those two compounds recommended by Korean, Chinese, European, and Japanese pharmacopoeia is biased against red ginseng preparations, particularly the extract, because of the relatively lower content of the two agents in red ginseng compared to white ginseng. In manufacturing fresh ginseng into red ginseng products, ginseng roots are exposed to high temperatures for many hours, and the naturally occurring ginsenoside $\mathrm{Rb} 1$ and $\mathrm{Rg} 1$ are converted to artifact ginsenosides such as $\mathrm{Rg} 3, \mathrm{Rg} 5, \mathrm{Rh} 1$, and $\mathrm{Rh} 2$ during the heating process. Analytical results of ginsenosides led us to set (20S)- and (20R)-ginsenoside $\mathrm{Rg} 3$, which are commercially available in the Korean market, as additional reference materials for ginseng preparations. In this respect, this study's purpose was to isolate high purity (20S)- and (20R)-ginsenoside Rg3 and develop those ginsenosides as standard reference materials by analyzing the full spectrum of physico-chemical properties of those two agents before establishing a validated analytical procedure.

We used red ginseng water extract to isolate artifact ginsenosides together with naturally occurring ginsenosides. Diaion HP-20 adsorption chromatography was employed for the preparation of crude saponin (CS). As we have reported previously [14], CS prepared by Diaion HP-20 demonstrated higher purity than obtained by conventional butanol partition chromatography. Then, we adopted silica gel flash chromatography for the crude fraction of ginsenosides. In that process we could 
roughly divide ginsenosides according to polarity. In the 2nd step of purification, a recrystallization technique was employed until we could obtain a white powder from (20S)- and (20R)-ginsenoside Rg3. Recrystallization technique plays a key role in the isolation and purification procedure for single ginsenosides as is the case with other natural compounds. In general, recrystallization is enough for the isolation of a single compound. However, in the case of ginsenosides, recrystallization does not guarantee its highest purity because in most cases diverse ginsenosides with similar chemical structures crystallize together. HPLC purification procedure is time-, labor-, and money-consuming, however, a preparative HPLC isolation procedure seems essential to obtain highest purity single ginsenosides unless alternative methods are devised. UV spectra of ginsenosides from other sources exhibited absorption pattern wavelengths other than 203 $\mathrm{nm}$, indicating poor purity. The commercially available ginsenosides showed a purity value of $92.5 \%$ when determined solely by HPLC method. European pharmacopoeia and Herbal Medicinal Products define that SRM employed for the quality control should be higher than $95 \%$ in purity when determined solely by HPLC. Therefore, those commercially available ginsenosides cannot meet the qualification as the SRM. In addition, those commercially available ginsenosides are not provided with the full spectrum of physico-chemical properties as an appendix. (20S)- and (20R)-ginsenoside Rg3 isolated from our laboratory revealed purity of $100 \%$ within the limits of HPLC. In addition, we prepared a full spectrum of physico-chemical data for (20S)- and (20R)-ginsenoside Rg3 as the SRM. Therefore, those ginsenosides can be employed as the SRM in GMP-based quality analysis.

Optical rotation for (20S)- and (20R)-ginsenoside Rg3 was analyzed in DMSO, pyridine and $\mathrm{MeOH}$ due to differences in solubility of the epimers. In addition, optical rotation values available from data base were found to be wrong. In the data base, optical rotation for (20R)ginsenoside $\mathrm{Rg} 3$ (c, 1.0 in $\mathrm{MeOH}$ ) was +8.5 . Actually, $(20 R)$-ginsenoside $\mathrm{Rg} 3$ is not soluble in $\mathrm{MeOH}$ at that concentration. We found the source of the value of +8.5 by searching the original reference. They proved the stereo-structure of (20S)-ginsenoside Rg3 by an organic synthesis method and reported the optical rotation for the compound as +8.5 (c, 1.0 in $\mathrm{MeOH}$ ). Our data for structure determination confirmed the stereochemistry of the two epimers. Although values of optical rotation for those epimers vary greatly depending upon the solvent, absolute values of plus or minus were not changed in the three organic solvents used in this experiment. The gap between +8.5 (value from reference) and +11.25 could be attributed to differences in sample purity and mechanical deviation. We are confident in the purity of the compounds that we isolated. Other spectrometric and purity data demonstrated no significant differences between ours and the reference values.

Recently, certified reference materials (CRM) of ginsenoside $\mathrm{Rb} 1$ and $\mathrm{Rg} 1$ were developed by the Korea Ginseng Corporation (Daejeon, Korea) in collaboration with the Korea Standard Research Institute (Daejeon, Korea). For a natural product, a CRM long term (3 yr) study period is required in close collaboration with qualified national standard institutes and traceability, stability, ash content, etc. should be investigated. Furthermore approximately $30 \mathrm{~g}$ of high purity single ginsenosides are required to establish the compound as CRM. It would be extremely time-, labor-, and money-consuming to obtain $30 \mathrm{~g}$ of natural reference material with $100 \%$ HPLC purity. The samples for spectrometric measurement can be recovered, but the samples for the determination of ash content and elemental analysis are not. Approximately 10 $\mathrm{g}$ of high purity ginsenosides would be burned to investigate ash content. Therefore, it may not be cost-effective to do quality analysis with CRM ginsenosides. Fortunately, reference materials for quality control analysis are not severely defined. According to Gaedcke et al. [6], GMP-based reference materials for quality control analysis general characteristics, identity, purity, and quantitation should be verified. In addition, the reference material should show HPLC purity higher than 95\%.

Taking all these circumstances into consideration, the (20S)- and (20R)-ginsenoside Rg3 standard reference materials isolated and documented by our research group meet all the requirements for a standard reference material for ICH-conforming GMP-based quality control. Validated analytical procedures established with those two standard reference materials together with (20S)- and (20R)-ginsenoside Rg3 will be presented elsewhere.

\section{ACKNOWLEDGEMENTS}

This study was supported by Konkuk University in 2012.

\section{REFERENCES}

1. Kim SK, Park JH: Trends in ginseng research in 2010. J Ginseng Res 2011; 35:389-398.

2. Kim DH: Chemical diversity of Panax ginseng, Panax 
quinquifolium, and Panax notoginseng. J Ginseng Res 2012; 36:1-15.

3. Han KD. The trends in chemical studies of ginseng. Korean J Ginseng Sci 1977;2:1-7.

4. Baek SH, Bae ON, Park JH: Recent methodology in ginseng analysis. J Ginseng Res 2012; 36:119-134.

5. International Conference on Harmonisation of Technical Requirements for Registration of Pharmaceuticals for $\mathrm{Hu}-$ man Use. Validation of analytical procedures: methodology. Geneva: The Conference, 1997.

6. Gaedcke F, Steinhoff B, Blasius H. Herbal medicinal products: scientific and regulatory basis for development, quality assurance and marketing authorisation. Stuttgart: Medpham, 2003.

7. Council of Europe. European pharmacopoeia. 6th ed. Strasbourg: Council of Europe, 2008.

8. Dong A, Ye M, Guo H, Zheng J, Guo D. Microbial transformation of ginsenoside $\mathrm{Rb} 1$ by Rhizopus stolonifer and Curvularia lunata. Biotechnol Lett 2003;25:339-344.

9. Teng R, Li H, Chen J, Wang D, He Y, Yang C. Complete assignment of ${ }^{1} \mathrm{H}$ and ${ }^{13} \mathrm{C}$ NMR data for nine protopanax- atriol glycosides. Magn Reson Chem 2002;40:483-488.

10. Wang W, Zhao Y, Rayburn ER, Hill DL, Wang H, Zhang $\mathrm{R}$. In vitro anti-cancer activity and structure-activity relationships of natural products isolated from fruits of Panax ginseng. Cancer Chemother Pharmacol 2007;59:589-601.

11. Cho JG, Lee MK, Lee JW, Park HJ, Lee DY, Lee YH, Yang DC, Baek NI. Physicochemical characterization and NMR assignments of ginsenosides Rb1, Rb2, Rc, and Rd isolated from Panax ginseng. J Ginseng Res 2010;34:113121.

12. Shao CJ, Xu JD, Kasai R, Tanaka O. Saponins from flower-buds of Panax ginseng cultivated at Jilin, China. Chem Pharm Bull 1989;37:1934-1935.

13. Su J, Li HZ, Yang CR. Studies on saponin constituents in roots of Panax quinquefolium. Zhongguo Zhong Yao Za Zhi 2003;28:830-833.

14. Kwak YS, Kyung JS, Kim SK, Wee JJ. An isolation of crude saponin from red-ginseng efflux by Diaion HP20 resin adsorption method. J Korean Soc Food Sci Nutr 2001;30:1-5. 